\title{
Editorial: Big Data Innovation for Sustainable Cognitive Computing
}

\author{
Anandakumar Haldorai ${ }^{1}$ - Arulmurugan Ramu ${ }^{2} \cdot$ Chee-Onn Chow $^{3}$ \\ Published online: 3 January 2019 \\ (C) Springer Science+Business Media, LLC, part of Springer Nature 2019
}

Cognitive Computing provides detailed guidance towards building a new class of systems that learn from experience and derive insights to unlock the value of big data. Cognitive computing is the creation of self learning systems that use data mining, pattern recognition and natural language processing to mirror the way the human brain works. The purpose of cognitive computing is to create computing systems that can solve complicated problems without constant human oversight. Today's cognitive computing solutions indicate an intelligent infrastructure that enables a new generation of customer and smart applications in all industries. Professionals who currently work with big data and analytics will see how cognitive computing builds on their foundation, and creates new opportunities. Cognitive Computing provides complete guidance to this new level of human machine interaction.

This special issue focused cognitive computing technologies, from knowledge representation techniques and natural language processing algorithms to dynamic learning approaches. We anticipate that the special issue will open new entrance for further research and technology improvements in this important area.

This special issue features six selected papers with high quality. The first article, "High Performance Reversible Direct Data Synthesizer for Radio Frequency Applications", proposed a new model for industrial, medical

Anandakumar Haldorai

anandakumar.psgtech@gmail.com

Arulmurugan Ramu

arulmr@gmail.com

Chee-Onn Chow

cochow@um.edu.my

1 Computer Science and Engineering, Sri Eshwar College of Engineering, Coimbatore, Tamil Nadu, India

2 Computer Science and Engineering, Presidency University, Bangalore, Karnataka, India

3 Electrical Engineering, Faculty of Engineering, University of Malaya, Kuala Lumpur, Malaysia and wireless communication networks simulate with a signal which has unknown frequency, phase and amplitude. They are frequently used for measuring the system response. Due to the increasing demands in communication area like 5G, the system response cannot be determined by using a single frequency. Hence a stable system which sweeps the frequency for the required range becomes mandatory. So many frequency generating elements, switching circuits were used in the past. The author researched that Direct Data Synthesizer (DDS) plays an adequate role in generating such stable sweeping frequencies. The proposed research work focus on highly demanding DDS with an active protecting circuit through reversible logic gate is evaluated.

The second work titled "Enhanced Energy in Sensors by Avoiding Voids and Saving Sensitive Data on Cloud using B + Tree Index with Retrieval of Query Predicates", presents the problems of storing sensor data on cloud and conserving in an amazing way. Diverse technology applications of cloud can be supported with efficient use of sensors. Sensors are defined as small hardware devices utilized in different applications. But the major issue with sensor is deprivation in battery. A sensor, which is not transmitting the packets, is eminent as void. In a network, voids are highly developing the energy and this leads to poor enactment. Avoiding void sensors along a path from source to destination will progress the energy efficiency. In this paper, a void avoidance mechanism is used with the exclusion of void nodes.

The third article with the title "Data Transmission Using IoT in Vehicular Ad-Hoc Networks in Smart City Congestion", the author proposes Smart City Congestion management system using IoT and Vehicular Ad-Hoc Networks. It also discusses the ways to minimize the limitations connected to IoT deployment and implementation in smart city environment using multi mediator scheme. The fundamental routing standards for vehicular ad hoc networks might adjust them to the autonomously altering transmitting conditions and recurrent breakages. Based on the analysis the intention is to validate the feasibilities to employ the mediator scheme as a brilliant 
solution for improving the routing schemes. The focus is to design a multi-mediator scheme for carrying out routing in VANETs. This fresh routing scheme is based on four mediators synchronized in locating optimal routes and in minimizing the traffic within the network.

The Fourth article focused "SMACLAD Secure Mobile Agent Based Cross Layer Attack Detection and Mitigation in Wireless Network", where the author proposes a new security framework to analyze and deal with cross layer attacks in wireless networks. The attack detection component is focused on bayesian learning detection scheme to detect the physical layer attacks and to overcome network, MAC layer and application layer attacks. A secure routing is proposed by creating server like wireless node. The proposed scheme helps to detect malicious, selfish and misbehaving node in wireless networks. The composed protocol is tested on a framework with cross layer attack that utilizes jamming and achieves preferred tradeoff between security and performance. The SMACLAD protocol performs better than the AODV_MALICIOUS and BLAD protocol in this aspect.

The Fifth article titled "Dominance Lagrange Optimized Rule Generation for Decision Table Evaluation", the work proposes Dominance Principle-based Reduct with Dominance Lagrange Optimized Rule Generation (DLO-RG) model the author analysis of obtained decision rule set. In DLO-RG, the degree of coherence to the dominance factor is defined. The major work classified and the coherence degrees of objects are combined. The combined values used to find the preference and relation dominance factor to generate the reducts of an input decision table. To the generated reducts, a Deterministic Global Optimization model using Lagrange is designed to optimize the rule generation. This method improves the rule extraction accuracy, reduces the rule generation time and number of rules generated through Lagrange formulation. Dominance Principle Reduct algorithm is used in a dynamic manner for reducing the rule generation time with the aid of measuring dominance factor.

The Last article titled "Effective Big Data Retrieval Using Deep Learning Modified Neural Networks", in the proposed technique executes general pre-processing extraction and closed recurrent item dataset to find the weight of provided data using entropy measure and frequent item measure. The cluster documents utilizing the k-means algorithm and then classifies using Deep Learning Modified Neural Networks (DLMNN). The proposed DLMNN weight parameters are optimized using the Cuckoo Search (CS) optimization algorithm. The comparison outcome illustrates shows that the proposed DLMNN technique has specified high precision, recall, together with F-score.
Acknowledgements The guest editors are thankful to our reviewers for their effort in reviewing the manuscripts. We also thank the Edit-in-Chief, Dr. Imrich Chlamtac for his supportive guidance during the entire process.

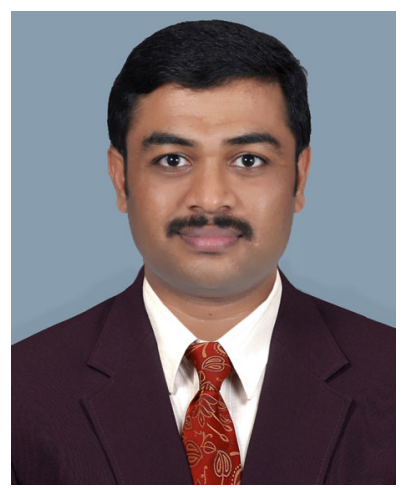

Anandakumar Haldorai, Professor (Associate) and Research Head in Department of Computer Science and Engineering, Sri Eshwar College of Engineering, Coimbatore, Tamilnadu, India. He has received his Master's in Software Engineering from PSG College of Technology, Coimbatore, $\mathrm{PhD}$ in In formation and Communication Engineering from PSG College of Technology under, Anna University, Chennai. His research areas include Cognitive Radio Networks, Mobile Communications and Networking Protocols. He has authored more than 52 research papers in reputed International Journals and IEEE, Springer Conferences. He has authored 5 books and many book chapters with reputed publishers such as Springer and IGI. He has served as Editor in Chief in Inderscience IJISC and reviewer for IEEE, IET, Springer, Inderscience and Elsevier journals. He is also the guest editor of many journals with Wiley, Springer, Elsevier, Inderscience, etc. He has been the General chair, Session Chair, and Panelist in several conferences. He is a senior member of IEEE, MIET, MACM and EAI research group.

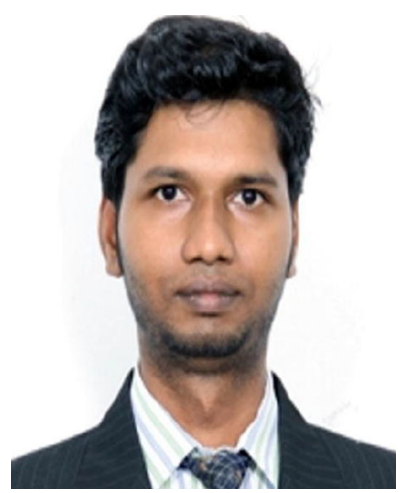

Arulmurugan Ramu, Professor in Department of Computer Science and Engineering, Presidency University, Bangalore, India. His research focuses on the automatic interpretation of images and related problems in machine learning and optimization. His main research interest is in vision, particularly high level visual recognition in computer vision, image and video classification, understanding and retrieval. Some of the most recent work is fundamental technological problems related to large-scale data, machine learning and artificial intelligence. He has authored more than 35 papers in computer vision and machine learning conferences and journals. He is the recipient of $\mathrm{PhD}$ degree in Information and Communication Engineering from Anna University, Chennai. 


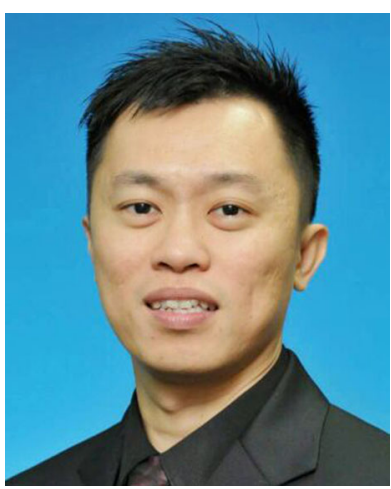

Chee-Onn Chow received his Bachelor of Engineering (Hons) and Master of Engineering Science degrees from University of Malaya, Malaysia in 1999 and 2001, respectively. He received his Doctorate of Engineering from the Tokai University, Japan in 2008 . He joined the Department of Electrical Engineering as tutor in 1999, and subsequently been offered a lecturer position in 2001. He is currently an Associate Professor in the same department since 2015. His research interests include various issues related to wireless communications. He is a Chartered Engineer (IET, UK), a Professor Engineer (BEM, Malaysia) and a Senior Members of IEEE. 